\title{
Adapted conservation measures are required to save the Iberian lynx in a changing climate
}

\author{
D. A. Fordham ', H. R. Akçakaya² , B. W. Brook', A. Rodríguez ${ }^{3}$, P. C. Alves ${ }^{4,5}$, E. Civantos ${ }^{4,6}$, \\ M. Triviño $0^{6,7}$, M. J. Watts ${ }^{1,8}$ and M. B. Araújo $0^{6,9,10 \star}$
}

The Iberian lynx (Lynx pardinus) has suffered severe population declines in the twentieth century and is now on the brink of extinction ${ }^{1}$. Climate change could further threaten the survival of the species ${ }^{2}$, but its forecast effects are being neglected in recovery plans ${ }^{3,4}$. Quantitative estimates of extinction risk under climate change have so far mostly relied on inferences from correlative projections of species' habitat shifts ${ }^{5}$. Here we use ecological niche models coupled to metapopulation simulations with source-sink dynamics ${ }^{6,7}$ to directly investigate the combined effects of climate change, prey availability and management intervention on the persistence of the Iberian lynx. Our approach is unique in that it explicitly models dynamic bi-trophic species interactions in a climate change setting. We show that anticipated climate change will rapidly and severely decrease lynx abundance and probably lead to its extinction in the wild within $\mathbf{5 0}$ years, even with strong global efforts to mitigate greenhouse gas emissions. In stark contrast, we also show that a carefully planned reintroduction programme, accounting for the effects of climate change, prey abundance and habitat connectivity, could avert extinction of the lynx this century. Our results demonstrate, for the first time, why considering prey availability, climate change and their interaction in models is important when designing policies to prevent future biodiversity loss.

The Iberian lynx is the world's most endangered cat. Its rangecontractedfrom $40,600 \mathrm{~km}{ }^{2}$ inthe1950stol,200 km $2005^{1,8}$ Recentountestimateonly250 Iberianlynxirthewild Thismajordeclineisassociatedcloselywithsharpreductions inEuropeanrabbit(Oryctolaguscuniculus)abundance $\quad{ }^{8}$,caused bymyxomatosisvirusinthe1950sand,morerecently,rabbit haemorrhagicdisease ${ }^{9}$ (RHD).Over-huntingofrabbitsandthe modificationandragmentationofitsmixedgrasslandandforest habitathasexacerbatedtheproblemsofpreyscarcity,because rabbitsccounformorÆhar $80 \%$ fberiarlynxonsumption Non-naturamortalityofberianlynxsuchasrappingpoaching androadkills, havefurtherreducedlynxpopulationnumbers OnlytwoIberianlynxpopulationspersistinthewildatpresent comparedvithinèrthe 990 s

Exsitucaptivebreeding programmeshave been launched to facilitate the reintroduction of a genetically diverse pool of Iberian lynx into suitable areas within their historical range $^{12}$.Simultaneously, managementeffortisbeingdirected towardsincreasingthecarryingcapacityofreintroductionsites throughhabitatrestoration relocatingrabbitsandimitingdirect anthropogenic-relatedatalities ${ }^{4,13}$ Althoughwell-financedffort toaverttheextinctionofthischarismaticspeciesisunderway ( $>€ 94$ milliorfundingsince 994) ${ }^{4}$ non-accountedhreatssuch as climate change and its influence on prey abundance, are not being consideredrecoverplans.

Here we provide the most comprehensive analysis of the likelyeffectsofclimatechangeyetforathreatenedvertebrate. So far, models used to investigate how climate change will

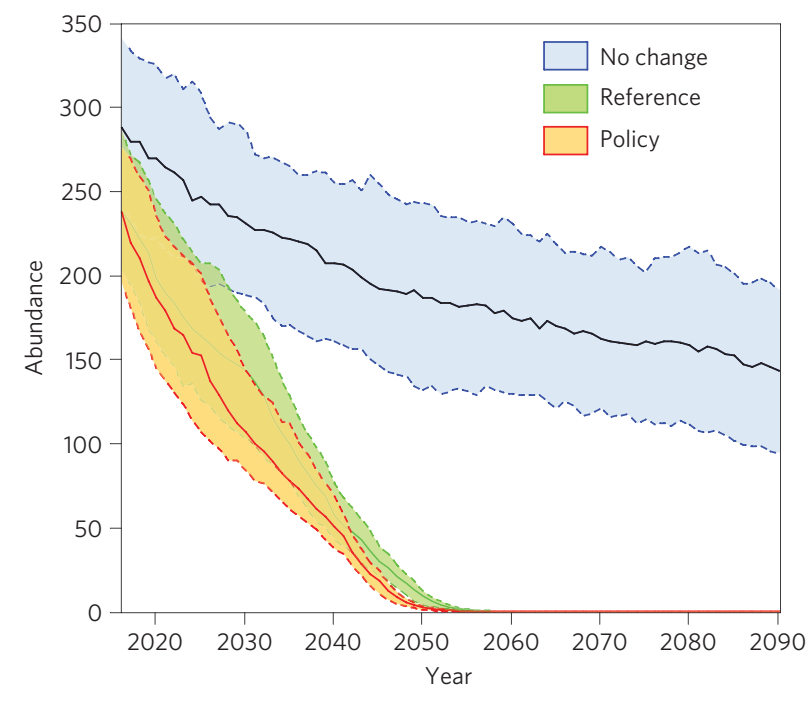

Figure 1 | Forecast lynx abundance in the Iberian Peninsula from 2015 to 2090 under three climate change scenarios. Scenarios are: no climate

10. change-where temperature and precipitation remains unchanged from the year 2000; high $\mathrm{CO}_{2}$ concentration Reference scenario (WRE750); and an

11. alternative low $\mathrm{CO}_{2}$ concentration Policy scenario that assumes strong mitigation of greenhouse gas emissions (LEV1). The solid lines show mean estimates for each scenario. Band widths represent 5th and 95th percentiles of population size derived from a stochastic metapopulation model with spatial habitat dynamics. Model variation characterizes demographic stochasticity, which is only one component of model uncertainty (see Supplementary Methods).

\footnotetext{
${ }^{1}$ Environment Institute and School of Earth and Environmental Sciences, University of Adelaide, North Terrace, South Australia 5005, Australia,

${ }^{2}$ Department of Ecology and Evolution, Stony Brook University, Stony Brook, New York 11794, USA, ${ }^{3}$ Department of Conservation Biology, Estación Biológica de Doñana, CSIC, Américo Vespucio s/n, 41092 Seville, Spain, ${ }^{4} \mathrm{CIBIO}$, Centro de Investigação em Biodiversidade e Recursos Genéticos \& Faculdade de Ciências, Universidade do Porto, 4485-661 Vairão, Portugal, ${ }^{5}$ Wildlife Biology Program, College of Forestry and Conservation, University of Montana, Montana 59812, USA, ${ }^{6}$ Department of Biogeography and Global Change, National Museum of Natural Sciences, CSIC, 28006 Madrid, Spain, ${ }^{7}$ Department of Biological and Environmental Sciences, University of Jyväskylä, PO Box 3540014 Jyväskylä, Finland, ${ }^{8}$ Information Technology Programme, AIS St Helens, PO Box 2995, Auckland 1140, New Zealand, ${ }^{9}$ Rui Nabeiro Biodiversity Chair, ClBIO, University of Évora, Évora, 7000, Portugal, ${ }^{10}$ Center for Macroecology, Evolution and Climate, University of Copenhagen, 2100 Copenhagen, Denmark. *e-mail: maraujo@mncn.csic.es
} 


\section{Table 1 | Extinction risk for the endangered Iberian lynx in the twenty-first century according to different climate and conservation scenarios.}

\begin{tabular}{lllll}
$\begin{array}{l}\text { Conservation } \\
\text { scenario }\end{array}$ & $\begin{array}{l}\text { Climate } \\
\text { scenario }\end{array}$ & EMA & $\begin{array}{l}\text { Probability of } \\
\text { extinction }\end{array}$ & $\begin{array}{l}\text { Median time } \\
\text { to extinction }\end{array}$ \\
\hline No extra manag. & No change & 58 & 12 & - \\
& Policy & 0 & 100 & 2051 \\
\multirow{2}{*}{ Extra manag. } & Reference & 0 & 100 & 2054 \\
\multirow{5}{*}{ Geopolitical } & Policy & 3 & 89 & 2066 \\
& Reference & 3 & 86 & 2065 \\
Peninsula-wide & Policy & 52 & 2 & - \\
& Reference & 52 & 4 & - \\
& Policy & 68 & 0 & - \\
\hline
\end{tabular}

Climate scenarios are a 'No change scenario' where temperature and precipitation remains unchanged from the year 2000, a stabilizing Policy scenario that assumes substantive mitigation of greenhouse gas emissions and a high $\mathrm{CO}_{2}$ concentration Reference scenario. Conservation scenarios are: no additional management intervention beyond what is occurring in Doñana and Sierra Morena (No extra manag.); an increase in active population management in potentially suitable habitat patches across the Iberian peninsula (Extra manag.); moving animals with an underlying aim to establish viable lynx breeding populations in every autonomous region within its recent historical range (Geopolitical); moving animals to areas of most favourable habitat across the entire lberian peninsula (Peninsula-wide). Extinction risk during this century is measured using expected minimum abundance (EMA), probability (\%) of total population size declining to zero (Probability of extinction) and median time to extinction (Median time to extinction)

affectbiodiversityatbroadergeographicalscaleshavefailedto accountexplicitlyfordynamifeedbacksofpeciesinteractions Weaddressthiskeylimitationusingcoupledecologicalnichepopulationmodelswithsource-sinkdynamics $\quad 6,7$,simulatingthe stochastic demographic responses of Iberian lynx to: spatial patterns ofrabbitabundanceconditionedbydisease,climateandlandusevariation;andchangesinclimatesuitabilityandlandscape modification.Weconsidertheinteractionbetweenrabbitsand Iberianlynxasunidirectional,becauselynxareextremelyrare, whereasrabbitsareabundantandhavegreaterthan30vertebrate predators, manyof themwidely distributedand locallyabundant Rare efforts to account for species interactions in climate-ecological forecasts have used overly simple approaches; by adding an interacting species as an additionalpredictor in a correlative model, orbyrestrictingthedistributionofonespeciestothemodelled distributionfhether ${ }^{16}$ Herewedirectlyaccounformportant per capita effects of rabbit abundance on the population growth rate of Iberian lynx in a climate change setting.

Weshowthatclimatechangeispredictedtohavearapid andseverenegativeinfluenceonlberianlynxabundance(Fig1), exceedingitsabilitytoadaptordispersetomoreclimatically favourableregionswherepreydensitiesaresufficienttosupport viablepopulations. Weestimatetimetoextinctiontobeless than50years(Table),evenwithrapidanddeepglobalcutsto anthropogenic greenhouse gas emissions (for instance, stabilization aarequivalenCO ${ }_{2}$ concentratiom 450 ppm(MiniCAMLE1; ref17))Wforecasthapopulationdeclinevilbccuntslightly fasterrateunderastrongmitigationemissionPolicyscenario (Fig1)causingslightlyshortemediartim๕œxtinction(2051 comparedto2054undertheReferencescenario;Tabld ).This apparentlycounter-intuitiveresultisbecausecoal-firedpower stationsalsoproduceatmosphericaerosolsthatsuppressglobal temperatureshroughdimming ${ }^{18}$ Replacingoakombustionwith cleanerenergyalternativesresultsinconditionsthatareinitially moreadverse(warmeranddrierbefore2050)forlberianlynx andrabbitsunderthePolicyscenariocomparedwithhigh-CO concentration Reference scenario (WRE750; ref. 19).

Intryingtœavertextinctionsinthewild,managershavdong consideredtheuseoffuman-assistedcolonizationofindividuals

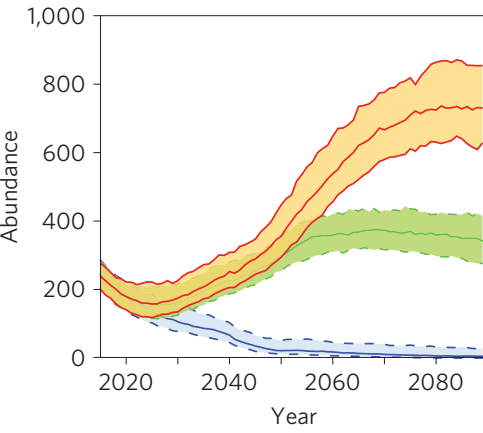

Present
Geopolitical
Peninsula-wide

b

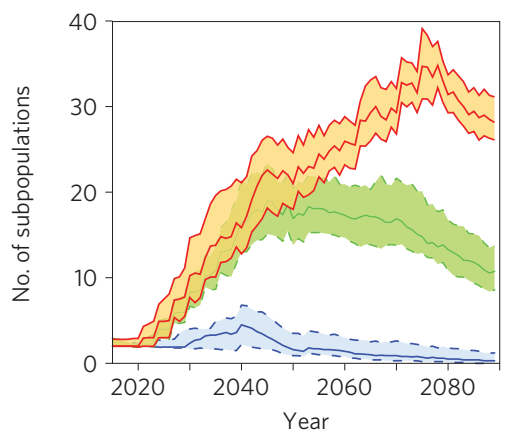

Figure 2 | The effect of management intervention on the persistence of Iberian lynx. a,b, Forecast lynx abundance (a) and number of populations

14. (b) in the Iberian Peninsula from 2015 to 2090 according to three possible management options. Forecasts are for a Policy climate scenario that assumes strong mitigation of greenhouse gas emissions (LEV1). The interventions are: present-day conservation practices, including increasing prey (lagomorph) densities, habitat alteration, preventing disease and non-natural mortality (Present); reintroducing captive-bred lynx to unoccupied habitat according to a Geopolitical scheme that favours establishing lynx populations in every autonomous region in Spain (Andalucía, Castilla-La Mancha, Comunidad Valenciana, Extremadura and Murcia), plus Portugal as an additional region, within its recent historical range (Geopolitical); and a strategy focused on releasing animals into the best-quality habitat regardless of region (Peninsula-wide). The solid lines show mean estimates for each scenario. Band widths represent 5 th and 95th percentiles, and characterize variation due to demographic stochasticity. They do not include error propagating from uncertainties in demographic parameters or forecasts of climate suitability (see Supplementary Methods).

tomoresuitablehabitat $\quad{ }^{20}$.However, as a climateadaptation strategy for Iberian lynx, such action has been criticized as inadequate if important factors relating to prey and habitat availability are ignored ${ }^{21}$. To avoid this simplification, we simulated areintroductionschemethataccountedsimultaneouslyforthe velocity,extentand nonlinearitiesinratesofclimate-induced changëhabitaqualityandheonnectivityprexavailabilityand physiologicakonditionsmportanttoberianlynx.Weshouthat ayearlyreleaseofsixanimals(agedbetweenland4years)of eachsexintohabitatpatchesrankedaccordingoacombination ofarryingapacity,nitiapopulatiorsizeçonnectivityandatøf survivahndecunditywouldaverthdikelyextinctionffberian lynxthiscentury(Fig2andTableandSupplementaryFigS1).

This reintroduction number is lower than that considered under the Iberian lynx reintroduction plan (release of 20-40 animals per year) and would not jeopardize the persistence of the breeding population (seépplementaryMethods).

Incontrast,weshowthatimplementingpresent-dayconservationpractices(increasingpreydensities, habitatmanagement, preventingdiseasendhon-naturamortality $\quad{ }^{1,4}$ atregionalevel 


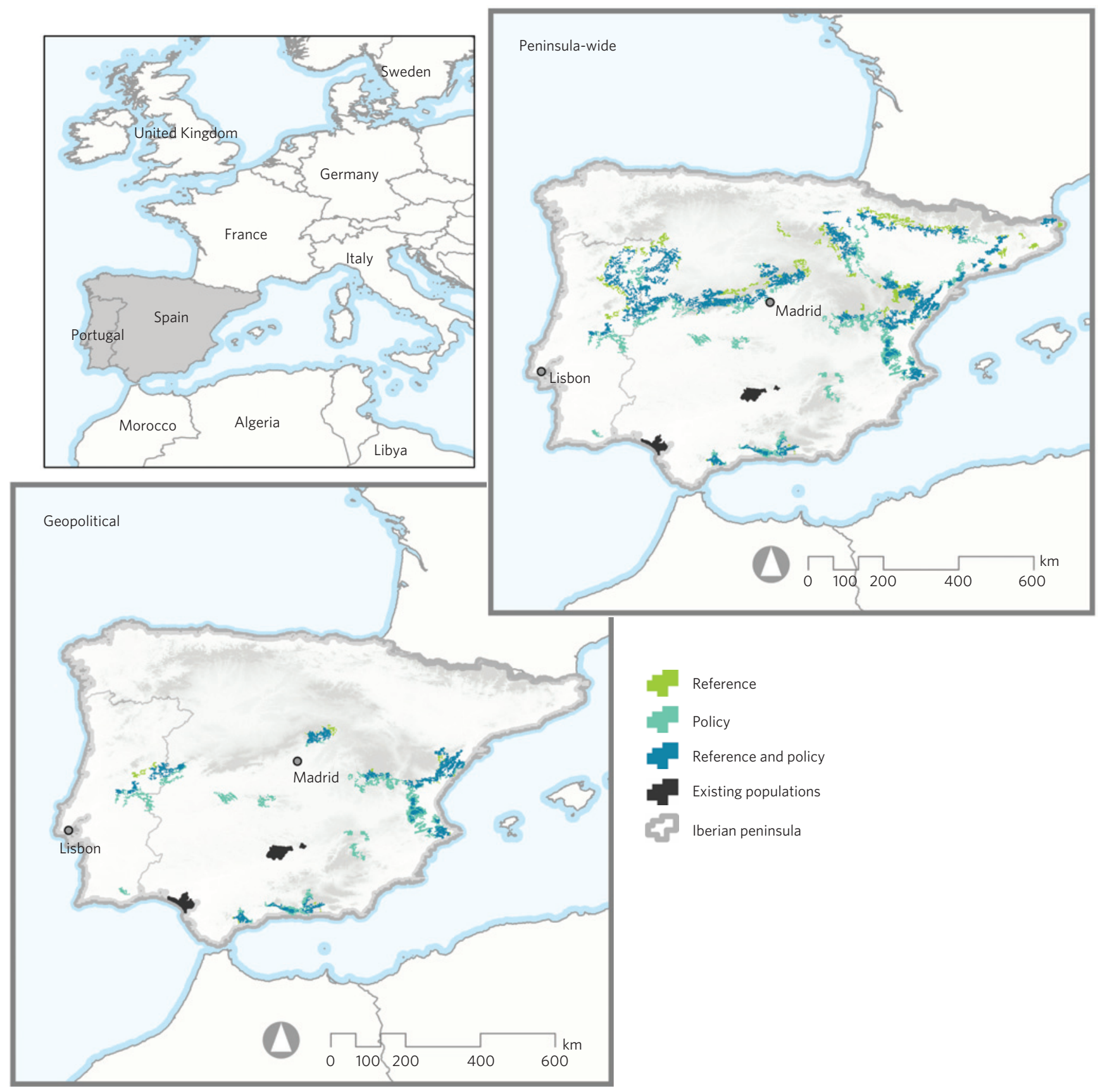

Figure 3 | Forecast location of lynx populations in the Iberian Peninsula in 2090. Occupied habitats are shown for the Peninsula-wide and Geopolitical reintroduction scenarios and two climate change scenarios: a high- $\mathrm{CO}_{2}$ concentration stabilizing Reference scenario (WRE750) and an alternative Policy scenario that assumes strong mitigation (LEV1). Maps capture lynx demographic responses to spatial patterns of rabbit abundance (conditioned by disease, climate and environmental variation) and changes in climate suitability and landscape modification. Only grid cells where lynx were present in $90 \%$ of stochastic iterations of the demographic model were treated as populated. See Supplementary Information and Methods for further details.

wouldonlyservÆodelaymediantimeæxtinctionby extensiveclearinganddestructionofnaturahabitatbyhumans hasdisruptedprocessesthatunderpinlberianlynxdispersaland establishment. We predict that $>40$ discrete areas of suitable habitat wouldbeavailableforpotentialcolonizationin2050,butinthe absenceofmanagedreintroductionsmostofthesewouldremain unoccupiedbecauseoflimited connectivitycausedbyhuman modificationfhłandscape.

Policymakers are considering the feasibility of establishing viableIberianlynxbreedingpopulationsineveryautonomous regionwithinitsecentistoricalange $\quad{ }^{22}$ Weshowhoweverthat
$<$ 15years, constrainingeintroduction₫œnlythosesuitablepatcheswithin therecenhistoricalang@fberianynx(SupplementaryFigS2), andspreadinganimalsevenlyacrossautonomousegionsusing Geopoliticalreintroductionstrategyresultsinfewerlberianlynx living in fewer populations in the future compared with a Peninsulawidereintroductionstrategywherebynimalsreeleasedntahe best-qualityhabitaregardlessfegion(Fig2anळupplementary FigS1).Wepredictfinalpopulationsizein2090tobebetween 190 and 275 animals (5thand95thpercentiles, respectively), approximatelythesameastoday'spopulationsize $\quad{ }^{4}$,livingin 7-10subpopulationfortheGeopoliticaleintroductionstrategy, comparedwith654-896animalsivingin25-31subpopulations 
forthePeninsula-widestrategy.Uncertaintiesintheseestimates areduetodemographicvariation,drivenbymultiplestochastic runsofthemetapopulationmodelandthecoupleddynamics oftherabbit-lynxbi-trophicinteraction. Theydonotinclude erropropagatingfromuncertaintiesindemographiqarameters or forecasts of climate suitability (see Supplementary Methods for a detailed description of model uncertainty). The reason for this large differenceishath Peninsula-widestrategyconsiderhabitatsin the northern half of Iberia as potential refugia from climate-induced shiftsinphysiologicalconditions,rabbitabundanceandhabitat availability (Fig3 and Supplementary Fig. S3). However, even then, anegativeimbalancebetweentherateofestablishmentofnew potentiarelocationsitesandhextirpationofldsexpectedto occuiforthePeninsula-widestrategyafter2065(Fig2).Thusin thefuturemanagersmightneedoconsidemovingberianlynx beyondtheirPleistocenerefugia(wheretheyevolved ${ }^{23}$ ) partsofEurope,wherethebiophysicalcharacteristicsofthenew locationbettematchtheed $\mathbf{s}$ fhespeciesndtstaplerey Forexample,fossildatashowthattheIberianlynxoritsnear relatives were distributed in southeastern France in the Pleistocene ${ }^{8}$.

Another potential management option is to improve the quality, connectivityandpermeability(ofthenon-habitatlandscapematrix)oflberianlynx'spresentandfuturehabitats,bydeveloping habitatorridorslonglimatpathways ${ }^{25}$ Howeverthemmediacyofheclimatehreafacedyiberiarlynx (highprobabilityof extinctionirthewildwithirthenex60yearsFig1)pairedwith boththetremendouscostassociatedwithestablishinglarge-scale movementorridors ${ }^{26}$ andhÆechnicafeasibilityofranslocating Iberianlynx ${ }^{4}$,impliesstronglythatthedispersalprocessesthat havebeendisruptedbylossofhabitatconnectivitycanbemost cost-effectivelyandquicklyrestoredusingarefullymanagedeintroductionsnotorridors 24 .

OurresultssuggestthatIberianlynxabundanceisdeclining (Figl,seealsoref.1).However,lynxabundanceatDoñana and Sierra Morena (the two extant populations) may have increasedinrecentyearsinresponsetointensivemanagement operations ${ }^{4}$.Recentconservationmeasureshaveincludedefforts talecreasenthropogenimortalityamonglispersingberianynx and broker conservation agreements with private landholders ${ }^{4}$, two managementactionsnotfullycapturedinourmodels, bothof whickoulфotentiallymfluencerajectorieopopulatiorgrowth in the absence of global warming. Our systematic sensitivity analysis (see Supplementary Information), which identified primary sources of demographic parameter uncertainty in model predictions, supportsthispremise. Althoughourforecastfinalmean and expectedminimumabundanceestimateforberianlynxproved tobemostsensitive to the frequency of outbreaks offeline leukaemiairus se@upplementaryMethods)lynxurvivalaged $\geq 2$ years) wasthesecondmostimportant parameterinthe model(SupplementaryTableS1).Althoughitremainsunclear whether management intervention in the two extant populations of IberianlynxinDoñanaandSierraMorenaisdefinitelyreversing populationdeclines ${ }^{1,4,22}$, present-dayeffortswillbeinsufficient forachievingthelong-termconservationoflberianlynx.Our dynamiametapopulationmodelresultsclearlyshowthathabitat connectivity today is inadequate to support sufficient range movementforaneffectivenatural-adaptationresponsetothe velocityofenvironmentalchangeforecastforIberianlynxthis century. Refining field estimates of Iberianlynxsurvival will strengthenmodelprecisionandshouldbeapriorityforfuture Iberiarlynxesearch.

The risk of extinction faced by Iberian lynx within the next50yearsishigh.Althoughexistingbreedingprogrammes and reintroduction plans promote the persistence of Iberian lynx(atleastin thenearterm), wehavedemonstrated that theimplementationofacarefullyplannedrelocationstrategy, accountingsimultaneouslyfortheffectsoflimatechangeprey availabilityconditionedbydisease,andhabitatconnectivity,will be crucial, irrespective of any global decision on mitigating greenhousegamissions.

\section{Methods}

Wersedouplediche-populationmodels $\quad{ }^{7}$ tosimultaneouslymodelberiarlynx anduropearrabbirangedynamicsndlimatehangeWeneratedannual timseriesoflimatechangdayerfonnuarainfalhndmeartemperatureof thettesandoolesmonthfotheriod2000-21001singegionallyskilful ensemble-averagedclimatforecasts ${ }^{27}$ Occurrencerecords sincel 950)were usedtfiecologicahichemodel(ENMs)separatelyforthdberianlynxand EuropearrabbitModelswerparameterizedorepresenpresentlimatic-habitat preferencesnd forecaspotentiafuturedistributionsTherationaleishat ENM-modelleduitabilityprovidessurrogat forpecies'carryingapacity capturingmoreharth physiologicakonstraintshatlefin $p r e s e n c e / a b s e n c e n t$ giverlocationwhichartherbersedndemographimodels fromalternativÆNMsanvarysubstantiallyunderthesameclimatechange scenariowecomputedevendifferenENMapproache\$BIOCLIMMahalanobis andEuclideandistancesgeneralizedineamodelsrandonforestmaximum entropyandGARP andbbtainedconsensuforecasbyassigningqualveights to a subset of skilful methods. We separately identified CORINE land-use categories (centredorthegea2000 appropriatfobreedinghabitafoiberiarlynxand rabbibccupancy(SupplementaryTables2an\$3)Adand-usemodification haseftstrongistoricalegacyorthedistributiomfberianlynxandabbits thisnformationwassectoconstraintheuitabilityoENMpredictionsboth foithpresent-dayanduture $\quad{ }^{29}$ Ouprojectionsssumehatynxandabbits wilkonserveheirclimatic-habitapreferencesithfutureandhatand-use remainspatiallyconstant.

Weuiltademographiprojectionforrabbitswithgrid-basedspatial structure,wherebyeachgridcellwasmodelledwithitsownscalar-stochastic modelThearryingapacityofabbitsineackelwabasedorENMredictions, scaledythemaximumandminimumrecordedannuarabbitbundanceper hectareThempactofnyxomatosismabbitbundancevamodelledmplicitly, whereasheimpactoRHDwasmodelledexplicitlyDiseasesweremodelled asemporallyuncorrelatedandon-interactivebecausempiricallatadirectly describingliseaseinteractionsndheireffectsorabbititaratescrosswide spectrumofabbitlensitiesrenoavailableTaccounforancertaintyirRHD estimates, 100abbimodelswerbuilteachwithdifferengridelfrequencies oRHDoccurrenceandeverity(samplefromwithirthdikelyappendower parameteibounds)andurforsingleterationundebothReferenceand PolicyCO ${ }_{2}$ concentrationscenarioandno-climatechangescenario,where temperaturean precipitatiorremainedinchangefromthear2000Using thesøutputs,wehemappedabbitdensityeachyear(2000-2100)foreach modelcapturingtochasticityininteractionbetweerabbitlemographydisease andource-sinkynamics.

Followingtherabbitmodellingstep,webuiltspatiallystructured metapopulationmodelfortheberianlynx,whereachsubpopulationwas representedwithsex-structuredstage-structuredstochastimodelThøizennd locationfubpopulationswageneratedısingNMpredictionsf̧orecastpatial patternsofabbiabundance (seprevioustep andarestimateofn-ground managementeffortThedensity-dependencesub-modelaccountedoirndividuals withandwithouterritoriesndmodifiedurvivahndecundityafunctions $\mathrm{f}$ rabbiandyndensityPopulation\$ocatedutsidprotectedareasweremodelled witharadditionall $0 \%$ fixedncreasemortalityDispersahccounteforthe interactiorbetweemovementatesndandoverWemodelledh probability of an outbreak of feline leukaemia virus as a per-population catastrophe.

Usingsingle-populationlynxmodelyecalculatedsustainablenumber of youngnimal\$agedetweenandtyears thatoulderemovedannuallffrom axtivbreedingopulatiomb0ynxWevaluatedandankedwhich atches toreleasdberiarlynxintœrthbasisofcompositemetridhataccounted foimportantecologicaprocessesReintroductionswerøpreadwhereossible, evenlyacrossutonomouregion\$ SupplementaryFigS2)foth Geopolitical strategy - targetinghemostuitableatchofabitaireachofwoutonomous regionsfothreeconsecutivegearswhereafothPeninsula-widestrategythe twdestankedhabitatsweressedegardlessprovincialocationVulnerability ofynxduringh 80 -yeainterva2010and2090wasmeasuredisingxpected minimumbundanceprobabilityofotapopulationsizedecliningazerand mediartimÆæxtinctiorfopersistenmoderunsAnnuabverageabundance andmetapopulationpatchoccupancywerealsausedtoprovidemeasure opopulationstability.

Thdevebfhecomplexityintherabbiandberiarlynxmodelseflected balancbetweentheneedomanagementactionsbbasedorrealistic modelshatdonotexcludemajobiologicaprocessesandmakinghemodel asobustapossibleaunderlyinguncertaintiesBayesiampproachesarbe usedœxploremodebrroimiche-populatiormodels $\quad{ }^{30}$ howeverusinghem topropagatancertaintyimomplexmodelssuchashdberiarlynxmodel, wouldecomputationallydemandingFurthermoreindependentevaluation 
ofuchmodelsixtremelydifficult $\quad{ }^{7}$ andbeyondhescopefhistudy. Wereportuncertaintiesinberiarlynxabundancederivedromtheinternal dynamicsofhestochastipopulationmodehndhecoupleddynamicsofhe rabbit-lynxrelationship.Weusedsensitivityanalysisodeterminhouthe rabbimodelwasensitivÆassumptionsurroundingliseasecarryingapacity andpopulationgrowthratesandwhetherassumptionsurroundingspatial and/omon-spatiallemographiparameterdargelyinfluencedbulynxmodel. Amoredetailedexplanatiomndustificatiom flatandmethodigiverirthe SupplementaryMethods.

\section{References}

1. Palomares, F., Rodríguez, A., Revilla, E., López-Bao, J. \& Calzada, J. Assessment ofheconservationeffort\$prevenextinctionfhdberiarlynxConserv. Biol. 25, 4-82011).

2. ThomasCDTranslocationfpeciesclimatehangeandhendfrying torecreat甲asecologicalommunitiesTrendEcolEvol. 26, 216-221(2011).

3. Gil-SánchezJM.Arenas-RojasR.García-TardíoM.Rodríguez-SilesJ\& Simón-MataMAHabitaassessmentcselecareaforeintroductionfhe endangeredberianynx.WildifBiolPract. 7, 1-192011).

4. Simón,MetalReverseofthedeclineofthendangerediberianlynx. ConservBiol. 26, 731-7362012).

5. Thomas,CDetalExtinctionrislfromclimatechangeNature 145-1482004)

6. KeithDAetPredictingxtinctionisksındeclimatehangeCoupling stochastipopulationmodelswithdynamibioclimatihabitatmodels. BiolLett. 4, 560-5632008).

7. Fordham,DA.Akçakaya,HR.Araújo,MB.,Keith,DA.\& Brook,BW.Toolforintegratingrangechangeextinctionriskand climatechangeinformationintoconservationmanagementEcography http://dx.doi.org/10.1111/j.1600-0587.2013.00147.x(2013).

8. RodríguezA\&DelibesMInternastructuren thegeographicangefhdberianynxEcography 25,314-3282002).

9. Delibes-MateosM.FerrerasP\&VillafuerteREuropearrabbipopulation trendsndssociatedactorsAreviewofhsituationith beriarPeninsula. MammaRev. 39, 124-1402009).

10.DelibesM.RodríguezA\&FerrerasPActionPlarfothConservationfhe Iberian Lnx Lynæardinus) Europe NaturandEnvironmenSeries,Vol. 111CouncibEuropPublishing2000).

11.RodríguezA\&DelibesMPatternsndausesfion-naturamortalityin thdberiarlynxduring 40 -yeaperiodbfangecontractionBiolConserv. 118, 151-161(2004).

12.Vargas, A. et al. The Iberian lynx Lynx pardinus conservation breeding program. IntZooYearbk 42, 190-1982008).

13.López-BaoJV.RodríguezA\&PalomaresFBehaviouraresponsœf trophispecialistthæberiarlynxţsupplementarfoodPatternofoodise andmplicationforonservationBiolConserv. 141, 1857-18672008).

14.Bellard,C.Bertelsmeier,C.LeadleyP.,Thuiller,W \&CourchampF. ImpactsoflimatechangeonthefutureofiodiversityEcolLett.

427, 365-3772012).

15.Delibes-Mateos\$.DelibesM.FerrerasP\&VillafuerteRKeyroløf EuropearrabbitsintheconservationfhewesternMediterranearbasin hotspotConservBiol. 22, 1106-11172008).

16.Kissling,WDetal.Towardsnovehpproachestomodellingbiotic interactionsmultispeciesssemblagestargepatialextentsJBiogeogr. 2163-21782012).

17.Wigley,TMLet UncertaintiesnclimatestabilizationClimatiChange 97, 85-121(2009).

18.Wigley,TMLCouldeducinfossil-fuebmissionsauseglobalvarming? Nature 349, 503-5061991).
19.WigleyTML.RichelsR\&EdmondsJAEconomiandenvironmental choicesithetabilizatiomftmosphericO 2 concentrationsNature 379, 240-243 1996).

20.GriffithB.ScottJM.CarpenterJW\&ReedCTranslocationsspecies conservationtoolStatusndtrategyScience 245, 477-4801989).

21.Vilà,M\&HulmePJurassidParkనNothanks.TrendEcolEvol. 497-4982011).

22.Rodríguez,A.CalzadaJ.RevillaE.López-BaoJV \&PalomaresF. BringingciencbacktheconservationfhdberianynxConservBiol. 737-7392012).

23.FerrerM\&NegroJJThenearextinctionfwdarg®uropearpredators: SupespecialistpaxpriceConservBiol. 18, 344-3492004).

24.Hoegh-GuldbergOetalAssistedolonizationandapidclimatechange. Science 321, 345-3462008).

25.AlagadorDetlLinkingikewithikeOptimisingonnectivity betweerenvironmentally-similahabitatsLandscapEcol. 27, 291-301(2012).

26.Fischlin,Aetinlmpacts,AdaptationandVulnerability(edParryML.) 211-272 CambridgdnivPress2007).

27.FordhamDA.Wigley,TML.,WattsMJ\&BrookBWStrengthening forecastsoflimatechangempactswithmulti-modeensembleaveraged projectionsısingMAGICC/SCENGEN.3Ecography 35, 4-82012).

28.VanDerWalJ.ShooLP.Johnson,CN\&Williams,SEAbundance andhenvironmentahicheEnvironmentakuitabilityestimatedrom nichemodelpredict\$heuppeitimiofocahbundanceAmNatur. 282-291(2009).

29.StantonJC.Pearson,RG.Horning,N.Ersts,P\&Akçakaya,HR. Combiningtatiandlynamiøariablesinpeciestistributiomodelsınder climatehangeMethodEcolEvol. 3, 349-3572012).

30.PagelJ\&s churrFMForecastingpeciesangebystatisticalstimationf ecologicahichesndpatiapopulationdynamicsGlobEcolBiogeogr. 293-3042012).

\section{Acknowledgements}

ThisesearchwasnitiatedrworkshopatheNationaMuseumoNaturabciences, MadridledbyM.B.AandfundedyFP6-MACISSSP/STREP/0778Australian ResearckouncilARC $\$$ upportedD.A.F.B.W.BandM.J.W(LP0989420DP1096427, FT100100200andFS110200051)NASANNX09AK19GandNSHDE-1146198 supportedH.R.A.FCTPTDC/AAC-AMB/98163/2008IntegratedProgramofC\&DT Nol/SAESCTN/ALENT-07-0224-FEDER-001755andDanishNSFupportedM.B.A.; SpanishesearchCounci(CSICgran200830I195 andheAndalusianDepartment ofnnovationanळcience(P06-RNM-01903)supportedA.REVirgósndCFerreira providedurtheexperadviceorrabbitdistributionandabundanceCMellimand NClisbygeneratedhfigures.

\section{Author contributions}

ThedesigmfhiprojectwasheresulbfliscussionsinvolvingllauthorsM.B.A

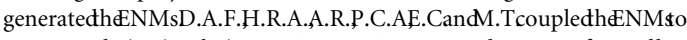
metapopulationsimulationsD.A.F.H.R.A.B.W.BandM.J.Wperformedhenalysis. D.A.FandM.B.AwrotÆhenitiallrafofhemanuscriptandlhuthorsontributed tohewritingfhefinalversionfh commentedrthemanuscript.

\section{Additional information}

Reprintsind permissions information is available online at www.nature.com/reprints. Correspondence and requests for materials should be addressed to M.B.A. 